\title{
SYNTHESIS AND ANTIFUNGAL ACTIVITY OF BENZOXAZOLE DERIVATIVES WITH THEIR SAR ANALYSIS BY SAS-MAP
}

\author{
KAMIAR ZOMORODIAN ${ }^{1}$, SOGHRA KHABNADIDEH ${ }^{2}$, AMIRHOSSEIN SAKHTEMAN ${ }^{2}$, BI \\ BI FATEMEH MIRJALILI ${ }^{3}$, MOHSEN RANJBAR ${ }^{2}$, LEILA ZAMANI ${ }^{2 *}$
}

${ }^{I}$ Department of Parasitology \& Mycology, School of Medicine, Shiraz University of Medical Sciences, Shiraz, I.R. Iran

${ }^{2}$ Faculty of Pharmacy and Pharmaceutical Sciences Research Centre, Shiraz University of Medical Sciences, Shiraz, I.R. Iran

${ }^{3}$ Department of Chemistry, College of Science, Yazd University, Yazd, P. O. Box 8915813149, Iran

*corresponding author: l.zamani2008@gmail.com

Manuscript received: October 2018

\begin{abstract}
A simple and efficient method has been developed for the synthesis of benzoxazole derivatives. In the first step, 2aminophenols reacted with various aldehydes in the presence of titanium supported nano-silica to produce an imine intermediate. Then the imine was oxidized to get the final azole compounds. Eleven derivatives were synthesized $(\boldsymbol{c})-(\boldsymbol{m})$ via this simple and environmentally friendly procedure with high yields. The simple methodology (only 2 steps) with high yields for the reactions and easy procedure are the advantages of the newly developed method. The identification and characterization of all the synthesized compounds were confirmed by melting point, thin layer chromatography, FT-IR, ${ }^{1} \mathrm{H}$ NMR and ${ }^{13} \mathrm{C}$ NMR spectral data. Also elemental analysis was applied for five compounds. All the compounds were screened for antimicrobial activity by broth microdilution methods as recommended by CLSI. Of the tested compounds 2 (2,4-dichlorophenyl)-1,3-benzoxazole $(\boldsymbol{g})$, and 2-(4-chlorophenyl)-1,3-benzoxazole $(\boldsymbol{f})$ inhibited the growth of all examined fungi, while 2-(4-nitrophenyl)-1,3-benzoxazole (c) exhibited inhibitory activities only against the tested yeasts.
\end{abstract}

\section{Rezumat}

A fost dezvoltată o metodă simplă și eficientă pentru sinteza derivaților de benzoxazol. În prima etapă, 2-aminofenolii au reacționat cu diferite aldehide, în prezența nano-silicei cu titan pentru a produce un intermediar iminic. Ulterior imina a fost oxidată pentru a obține azoli finali. Au fost sintetizați cu randamente ridicate, prin această procedură simplă şi ecologică, unsprezece compuşi. Identificarea şi caracterizarea tuturor compuşilor sintetizați au fost confirmate prin: punctul de topire, cromatografie în strat subțire, FT-IR, ${ }^{1} \mathrm{H}$ RMN și ${ }^{13} \mathrm{C}$ RMN. De asemenea, analiza elementală a fost aplicată pentru cinci compuși. Toți compușii au fost analizați din punct de vedere microbiologic prin metode microdiluțiilor. Dintre compușii testați, 2-(2,4-diclorofenil)-1,3-benzoxazol ( $g$ ) și 2-(4-clorfenil)-1,3-benzoxazol $(f)$ au inhibat creșterea tuturor ciupercilor examinate, în timp ce 2-(4-nitrofenil)-1,3-benzoxazol (c) a prezentat activităţi inhibitoare numai împotriva drojdiilor testate.

Keywords: benzoxazole, synthesis, antifungal, $\mathrm{SAR}$ analysis, nano- $\mathrm{TiCl}_{4} \cdot \mathrm{SiO}_{2}$

\section{Introduction}

Systemic fungal infections increased dramatically in the past few decades, especially in immune-compromised individuals suffering from tuberculosis, cancer, AIDS, and in organ transplant recipients [1]. The widespread use of antifungal drugs and their resistance against fungal infections have led to serious health concerns. Although different drugs such as novel azole compounds are available for the treatment of superficial and systemic mycoses, they are not completely effective in all cases. In addition, they all possess a certain degree of toxicity and quickly develop resistance due to the large-scale usage. Therefore, an urgent need for new antifungal chemical structures as alternative agents to the existing ones is required [2].

In this regard, the azole ring system is present in biologically active compounds which possess high antifungal properties.
In our previous reports, we described the preparation procedure for a number of imidazole, benzimidazole and benzotriazole derivatives with biological interest [3-6]. In the current study, we have synthesized some new derivatives of benzoxazoles as antifungal agents. Usually, nitrogen and sulphur containing organic compounds display a wide range of biological activities. For example benzoxazole heterocycles display a broad spectrum of biological activities such as hypoglycaemic [7], antiulcer [8], antifungal [9], anticonvulsant [10], antitumor [11] and anti-inflammatory [12] activities. Different protocols for the synthesis of benzoxazoles have been developed including copper-catalysed cyclizations of $o$-halobenzanilides or cross-coupling of primary amides $[13,14]$ and direct condensation of $o$-aminophenol with carboxylic acid or aldehyde [15]. In most cases, more than one step is required to synthesize these heterocycles. To avoid these limitations, 
our study aimed towards the development of more efficient methods accompanied with higher yields for the synthesis of benzoxazoles in the presence of nano$\mathrm{TiCl}_{4} \cdot \mathrm{SiO}_{2}$. In our previous works we introduced titanium supported nano-silica as an environmental friendly and economical catalyst for organic reactions [16-21]. Easy handling, inexpensive, efficient, nontoxic, recoverable, and its reusability make this catalyst eco-friendly, synthetically acceptable and economically feasible. In the present study, we used this catalyst to synthesize our target compounds in a short period of time and with high yields in only two steps.

In order to compare the synthesized compounds based on theirs structures and activities, SAS (structure activity similarity) map analysis was performed. Furthermore, broth microdilution methods were used for screening the antifungal and antibacterial activities of synthetic compounds.

\section{Materials and Methods}

\section{Chemistry}

The chemicals were purchased from Merck and used without any additional purification. The products were characterized by FT-IR (ATR), ${ }^{1} \mathrm{H}-\mathrm{NMR}$, and a comparison of their physical properties with those reported in the literature was performed. FT-IR (ATR) spectra were acquired on a Bruker, Eqinox 55 spectrometer. A Bruker (DRX-400 Avance) NMR was used to record the ${ }^{1} \mathrm{H}$ NMR and ${ }^{13} \mathrm{C}$ NMR spectra. The melting points were measured by a Buchi melting point B-540 apparatus. The elemental analysis was done by a Costech ECS 4010 CHNS-O analyser.

General procedure for synthesis of benzoxazole derivatives $(\boldsymbol{a})-(\boldsymbol{m})$

A mixture of 2-aminophenol derivatives $(1.5 \mathrm{mmol})$ and substituted aldehydes ( $1 \mathrm{mmol})$ with Ti supported nano- $\mathrm{SiO}_{2}(0.1 \mathrm{~g})$ in $\mathrm{EtOH}(2 \mathrm{~mL})$ was stirred at room temperature for $1.5 \mathrm{~h}$. Following completion of the reaction, the solvent was filtered and evaporated; small amounts of cold water were added and then dried. Our suggestion was that an imine intermediate is the product of this step. To make sure about the formation of this intermediate we isolated and identified the product of this step only for two compounds (intermediates $(\boldsymbol{a})$ and $(\boldsymbol{b})$ for compounds $(\boldsymbol{c})$ and $\mathrm{d}$ respectively). Spectroscopic data confirmed our suggestion. Thus for other compounds $(\boldsymbol{e})-(\boldsymbol{m})$ the reaction was performed in situ without any separation of the intermediate.

Subsequently, in the next step, by oxidation of the imine intermediate via $\mathrm{KMnO}_{4}(1.7 \mathrm{mmol})$ and $\mathrm{CH}_{3} \mathrm{COOH}(0.08 \mathrm{~mL})$ in grinding, crude benzoxazole was formed. Purification of the crude compound was carried out by recrystallization from acetone (solid products) or by chromatography using silica gel and mixtures of $n$-hexane/ethyl acetate (70/30) of increasing polarity.

Table I

Spectral data of benzoxazole derivatives

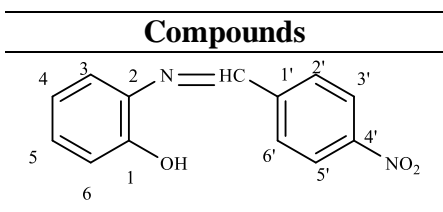

$\boldsymbol{a}$<smiles>O=[N+]([O-])c1cccc(C=Nc2ccccc2O)c1</smiles>

$\boldsymbol{b}$

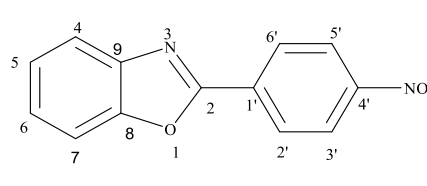

$c$

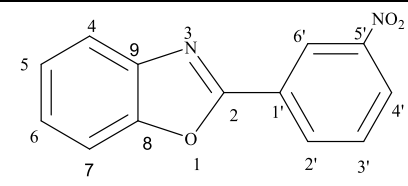

$d$

Spectral data Yield: 98\%, Yellow Solid, $\mathrm{Mp}=160-161^{\circ} \mathrm{C} ; \mathrm{FT}-\mathrm{IR},(\mathrm{ATR})=\overline{\mathrm{v}}: 3365(\mathrm{O}-\mathrm{H}$ stretch), 1587 (C=N stretch), 1509 ( $\mathrm{NO}_{2}$ asym. stretch), 1480 (C=C stretch), 1335 ( $\mathrm{NO}_{2}$ sym. stretch), 1287 (C-N stretch), 1241 (C-O stretch), $\mathrm{cm}^{-1},{ }_{1}^{1} \mathrm{H}$ NMR (400 MHz, CDCl $)$, $\sigma(\mathrm{ppm})=6.95\left(\mathrm{t}, J=8 \mathrm{~Hz}, 1 \mathrm{H}_{6}\right), 7.06\left(\mathrm{~d}, J=8 \mathrm{~Hz}, 1 \mathrm{H}_{4}\right), 7.18(\mathrm{brs}, 1 \mathrm{H}, \mathrm{OH}), 7.37(\mathrm{~d}, J=8$ $\left.\mathrm{Hz}, 2 \mathrm{H}_{3,5}\right), 8.10\left(\mathrm{~d}, J=8 \mathrm{~Hz}, 2 \mathrm{H}_{2}, 6^{\prime}\right), 8.37$ (d, $\left.J=8 \mathrm{~Hz}, 2 \mathrm{H}_{3},{ }^{\prime}\right), 8.8(\mathrm{~s}, 1 \mathrm{H},=\mathrm{CH}) ;{ }^{13} \mathrm{C}$ NMR $(300 \mathrm{MHz}, \mathrm{DMSO}), \sigma(\mathrm{ppm})=163.7(=\mathrm{C}), 144.2\left(\mathrm{C}_{1}\right), 133.9\left(\mathrm{C}_{1}\right), 130.9\left(\mathrm{C}_{5}\right)$, 127.4 $\left(\mathrm{C}_{6}{ }^{\prime}\right), 127.3\left(\mathrm{C}_{2}{ }^{\prime}\right), 123.1\left(\mathrm{C}_{5},\right), 121.7\left(\mathrm{C}_{3}{ }^{\prime}\right), 120.3\left(\mathrm{C}_{4}\right), 116.0\left(\mathrm{C}_{3}\right), 114.4\left(\mathrm{C}_{6}\right)$.

Yield: $86 \%$, Yellow Solid, Mp $=136-137^{\circ} \mathrm{C} ;$ FT-IR, $($ ATR $)=\bar{v}: 3345($ O-H stretch), 3046 (C-H stretch), 1630 (C=N stretch), 1583, 1476 (C=C stretch), $1513\left(\mathrm{NO}_{2}\right.$ asym. stretch), 1345 ( $\mathrm{NO}_{2}$ sym. stretch), 1286 (C-N stretch), 1239 (C-O stretch) cm ${ }^{-1},{ }^{1} \mathrm{H}$ NMR $\left(400 \mathrm{MHz}\right.$, Acetone), $\sigma(\mathrm{ppm})=6.8-7.0\left(\mathrm{~m}, 1 \mathrm{H}_{6}, \mathrm{OH}\right), 7.2\left(\mathrm{t}, 1 \mathrm{H}_{4}\right), 7.5\left(\mathrm{~d}, 1 \mathrm{H}_{3}\right), 7.85$ $\left(\mathrm{m}, 1 \mathrm{H}_{5}\right), 8.3-8.4\left(\mathrm{~m}, 2 \mathrm{H}_{4}{ }^{\prime}, 6^{\prime}\right), 8.5\left(\mathrm{~d}, 1 \mathrm{H}_{5},\right), 8.8\left(\mathrm{~s}, 1 \mathrm{H}_{2}{ }^{\prime}\right), 9.04(\mathrm{~s}, 1 \mathrm{H},=\mathrm{CH}) ;{ }^{13} \mathrm{C} \mathrm{NMR}$ $(300 \mathrm{MHz}, \mathrm{DMSO}), \sigma(\mathrm{ppm})=164.9(=\mathrm{C}), 145.4\left(\mathrm{C}_{1}\right), 139.7\left(\mathrm{C}_{3}{ }^{\prime}\right), 131.2\left(\mathrm{C}_{1}{ }^{\prime}\right), 129.6\left(\mathrm{C}_{6}{ }^{\prime}\right)$, 128.7 $\left(\mathrm{C}_{5}{ }^{\prime}\right), 126.1\left(\mathrm{C}_{5}\right), 131.2\left(\mathrm{C}_{4}{ }^{*}\right), 123.1\left(\mathrm{C}_{2}{ }^{*}\right), 122.4 .0\left(\mathrm{C}_{4}\right), 117.0\left(\mathrm{C}_{3}\right), 116.1\left(\mathrm{C}_{6}\right)$.

Yield: $92 \%$, Brown Solid, $\mathrm{Mp}=262-264^{\circ} \mathrm{C}\left[266-268^{\circ} \mathrm{C}\right]$ [25]; FT-IR, (ATR) = v: 3112 (C-H stretch), 1598 (C=N stretch), 1555, 1451 (C=C stretch), 1519 ( $\mathrm{NO}_{2}$, asym. stretch), $1348\left(\mathrm{NO}_{2}\right.$, sym. stretch) $\mathrm{cm}^{-1},{ }^{1} \mathrm{H} \mathrm{NMR}\left(400 \mathrm{MHz}, \mathrm{CDCl}_{3}\right), \sigma(\mathrm{ppm})=7.26-8.1(\mathrm{~m}$, $\left.4 \mathrm{H}_{4,5,6,7}\right), 8.44$ (brs, 4H $\left.6^{\prime}, 5^{\prime}, 3^{\prime}, 2^{\prime}\right)$; ${ }^{13} \mathrm{C} \mathrm{NMR}(300 \mathrm{MHz}, \mathrm{DMSO}), \sigma(\mathrm{ppm})=145.9\left(\mathrm{C}_{2}\right)$, 133.0( $\left.\mathrm{C}_{4}{ }^{\prime}\right), 132.9\left(\mathrm{C}_{1}{ }^{\prime}\right), 129.2\left(\mathrm{C}_{6}{ }^{\prime}\right), 129.1\left(\mathrm{C}_{2}{ }^{\prime}\right), 124.1\left(\mathrm{C}_{5}{ }^{\prime}\right), 123.0\left(\mathrm{C}_{3}{ }^{\prime}\right), 117.0\left(\mathrm{C}_{5}\right), 116.2\left(\mathrm{C}_{6}\right)$, $116.0\left(\mathrm{C}_{4}\right), 115.7\left(\mathrm{C}_{7}\right) \mathrm{ppm}$.

Yield: $87 \%$, Gray Solid, $\mathrm{Mp}=210-212^{\circ} \mathrm{C}\left[211-212^{\circ} \mathrm{C}\right]$ [25]; FT-IR, (ATR) $=\bar{v}: 3097$ (C-H stretch), 1613 (C=N stretch), 1525 ( $\mathrm{NO}_{2}$, asym. stretch), 1474, 1453 (C=C stretch), 1351 ( $\mathrm{NO}_{2}$, asym. stretch), 1241 (C-O stretch) $\mathrm{cm}^{-1},{ }^{1} \mathrm{H}$ NMR (400 MHz, Acetone),

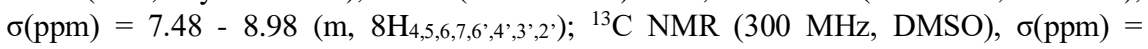
144.2 $\left(\mathrm{C}_{2}\right), 133.9\left(\mathrm{C}_{5}{ }^{\prime}\right), 130.9\left(\mathrm{C}_{2}{ }^{\prime}\right), 127.4\left(\mathrm{C}_{3}{ }^{\prime}\right), 127.3\left(\mathrm{C}_{5}\right), 123.1\left(\mathrm{C}_{6}\right), 121.7\left(\mathrm{C}_{4}{ }^{\prime}\right), 116.0\left(\mathrm{C}_{4}\right)$, $114.4\left(\mathrm{C}_{7}\right)$. 
FARMACIA, 2020, Vol. 68, 1

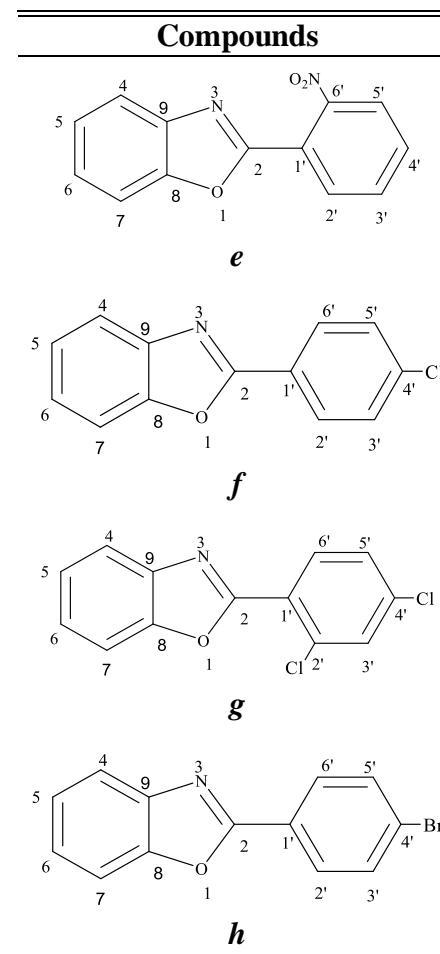

Spectral data

Yield: $90 \%$, Brown Solid, $\mathrm{Mp}=104-105^{\circ} \mathrm{C}\left[101-103^{\circ} \mathrm{C}\right]$ [25]; FT-IR, $(\mathrm{ATR})=\overline{\mathrm{v}}: 1620$

$\left(\mathrm{C}=\mathrm{N}\right.$ stretch), $1534\left(\mathrm{NO}_{2}\right.$, asym. stretch), $1452(\mathrm{C}=\mathrm{C}$ stretch $), 1349\left(\mathrm{NO}_{2}\right.$, sym. stretch),

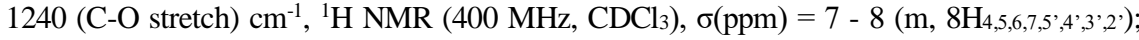
${ }^{13} \mathrm{C}$ NMR $(300 \mathrm{MHz}, \mathrm{DMSO}), \sigma(\mathrm{ppm})=145.4\left(\mathrm{C}_{2}\right), 133.0\left(\mathrm{C}_{6}{ }^{\prime}\right), 132.9\left(\mathrm{C}_{1}\right), 129.2\left(\mathrm{C}_{3^{\prime}}\right)$, 129.1 $\left(\mathrm{C}_{4}{ }^{\prime}\right), 128.7\left(\mathrm{C}_{2}{ }^{\prime}\right), 124.1\left(\mathrm{C}_{5}\right), 123.0\left(\mathrm{C}_{6}\right), 116.0\left(\mathrm{C}_{4}\right), 115.7\left(\mathrm{C}_{7}\right) ; \mathrm{C}_{13} \mathrm{H}_{8} \mathrm{~N}_{2} \mathrm{O}_{3}$, Calculated: C, 65.00; H, 3.36; N, 11.66. Found: C, 64.6; H, 3.39; N, 11.67\%.

Yield: 85\%, Brown Solid, $\mathrm{Mp}=145-147^{\circ} \mathrm{C}\left[148-150^{\circ} \mathrm{C}\right]$ [24]; FT-IR, (ATR) $=\bar{v}: 3060$ (C-H stretch), 1617 (C=N stretch), 1574, 1483 (C=C stretch), 1344 (C-N stretch), 1244 (C-O stretch), 1091 (C-Cl stretch) $\mathrm{cm}^{-1},{ }^{1} \mathrm{H}$ NMR (400 MHz, Acetone), $\sigma(\mathrm{ppm})=7.44-$

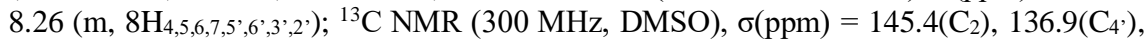
133.7 $\left(\mathrm{C}_{1}{ }^{\prime}\right), 129.6\left(\mathrm{C}_{6^{\prime}}\right), 129.1\left(\mathrm{C}_{2}\right), 128.8\left(\mathrm{C}_{5}{ }^{\prime}\right), 127.6\left(\mathrm{C}_{3^{\prime}}\right)$, 127.0( $\left.\mathrm{C}_{5}\right), 124.1\left(\mathrm{C}_{6}\right), 123.0\left(\mathrm{C}_{4}\right)$, $116.3\left(\mathrm{C}_{7}\right)$.

Yield: $88 \%$, Gray Solid, $\mathrm{Mp}=141-142^{\circ} \mathrm{C}\left[144-145^{\circ} \mathrm{C}\right]$ [24]; FT-IR, $($ ATR $)=\bar{v}: 3066$ (C-H stretch), 1590 (C=N stretch), 1560, 1471 (C=C stretch), 1375 (C-N stretch), 1191 (C-O stretch), 1027 (C-Cl stretch) $\mathrm{cm}^{-1},{ }^{1} \mathrm{H}$ NMR (400 MHz, Acetone), $\sigma(\mathrm{ppm})=7.45$

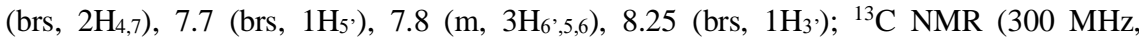
DMSO $), \sigma(\mathrm{ppm})=145.3\left(\mathrm{C}_{2}\right), 135.4\left(\mathrm{C}_{4}{ }^{\circ}\right), 128.8\left(\mathrm{C}_{2}{ }^{\prime}\right), 128.7\left(\mathrm{C}_{1^{\prime}}\right), 127.4\left(\mathrm{C}_{6}{ }^{\circ}\right), 126.6\left(\mathrm{C}_{3^{\prime}}\right)$, 124.0 $\left(\mathrm{C}_{5}\right), 122.8\left(\mathrm{C}_{6}\right), 116.9\left(\mathrm{C}_{4}\right), 115.7\left(\mathrm{C}_{7}\right)$.

Yield: $91 \%$, Gray Solid, $\mathrm{Mp}=154-156^{\circ} \mathrm{C}\left[157-158^{\circ} \mathrm{C}\right]$ [24]; FT-IR, $(\mathrm{ATR})=\overline{\mathrm{v}}$ : 3057(C-H stretch), 1616 (C=N stretch), 1592, 1483 (C=C stretch), 1294 (C-N stretch), 1243 (C-O stretch), 1068 (C-Br stretch) $\mathrm{cm}^{-1},{ }^{1} \mathrm{H}$ NMR (400 MHz, DMSO- $\left.d_{6}\right), \sigma(\mathrm{ppm})=$

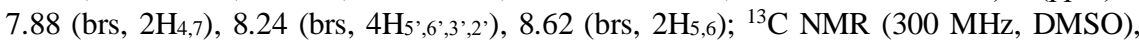
$\sigma(\mathrm{ppm})=144.4\left(\mathrm{C}_{2}\right), 132.6\left(\mathrm{C}_{5^{\circ}}\right), 132.5\left(\mathrm{C}_{3^{\prime}}\right), 130.0\left(\mathrm{C}_{1^{\prime}}\right), 129.2\left(\mathrm{C}_{2}{ }^{\prime}\right), 129.1\left(\mathrm{C}_{6}{ }^{\prime}\right), 126.7\left(\mathrm{C}_{5}\right)$, 123.6 $\left(\mathrm{C}_{4}\right), 118.4\left(\mathrm{C}_{6}\right), 116.1\left(\mathrm{C}_{4}\right), 115.6\left(\mathrm{C}_{7}\right)$.

Yield: 68\%, Gray Solid, $\mathrm{Mp}=169-171^{\circ} \mathrm{C}$; FT-IR, $(\mathrm{ATR})=\bar{v}$ : $3163(\mathrm{C}-\mathrm{H}$ stretch$), 2955$ (C-H stretch), 1726 (C=O stretch), 1605 (C=N stretch), 1557, 1454 (C=C stretch), 1276 $\left(\mathrm{C}-\mathrm{O}\right.$ stretch) $\mathrm{cm}^{-1},{ }^{1} \mathrm{H}$ NMR $\left(400 \mathrm{MHz}, \mathrm{DMSO}-d_{6}\right), \sigma(\mathrm{ppm})=4.35\left(\mathrm{~s}, 3 \mathrm{H}, \mathrm{CH}_{3}\right), 7.92$

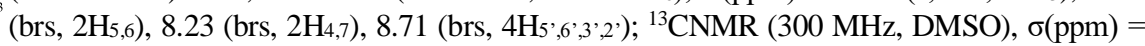
164.9 $(\mathrm{C}=\mathrm{O}), 145.4\left(\mathrm{C}_{2}\right), 133.7\left(\mathrm{C}_{9}\right), 131.2\left(\mathrm{C}_{1}{ }^{\prime}\right), 130.6\left(\mathrm{C}_{4}{ }^{\prime}\right), 129.8\left(\mathrm{C}_{5}{ }^{\prime}\right), 128.7\left(\mathrm{C}_{3^{\prime}}\right), 126.1\left(\mathrm{C}_{5}{ }^{\prime}\right)$, 124.2( $\left(\mathrm{C}_{2}{ }^{\prime}\right), 123.1\left(\mathrm{C}_{5}{ }^{\prime}\right), 122.4\left(\mathrm{C}_{6}{ }^{\prime}\right), 117.0\left(\mathrm{C}_{4}\right), 116.1\left(\mathrm{C}_{7}\right), 43.4\left(\mathrm{CH}_{3}\right) ; \mathrm{C}_{15} \mathrm{H}_{11} \mathrm{NO}_{3}$, Calculated: C, 71.14; H, 4.38; N, 5.53. Found: C, 70.64; H, 4.42; N, 5.89\%.

Yield: 83\%, Yellow Solid, $\mathrm{Mp}=220-222^{\circ} \mathrm{C}$; FT-IR, $(\mathrm{ATR})=\bar{v}: 3093(\mathrm{C}-\mathrm{H}$ stretch), $1602\left(\mathrm{C}=\mathrm{N}\right.$ stretch), 1552, $1453(\mathrm{C}=\mathrm{C}$ stretch$), 1519\left(\mathrm{NO}_{2}\right.$, asym. stretch), $1348\left(\mathrm{NO}_{2}\right.$,<smiles>COc1ccc(Cl)cc1-c1nc2cc(Cl)ccc2o1</smiles>
sym. stretch), 1285 (C-O stretch), 1063 (C-Cl stretch) $\mathrm{cm}^{-1},{ }_{1}^{1} \mathrm{H}$ NMR (400 MHz, Acetone),

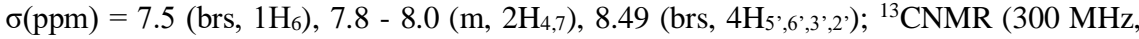
DMSO), $\sigma(\mathrm{ppm})=145.4\left(\mathrm{C}_{2}\right), 128.7\left(\mathrm{C}_{6},\right), 123.8\left(\mathrm{C}_{5},\right), 123.1\left(\mathrm{C}_{6}\right), 117.0\left(\mathrm{C}_{4}\right), 115.7\left(\mathrm{C}_{7}\right)$; $\mathrm{C}_{13} \mathrm{H}_{7} \mathrm{ClN}_{2} \mathrm{O}_{3}$, Calculated: C, 56.85; H, 2.57; N, 10.20. Found: C, 56.75; H, 2.62; N, $10.25 \%$.

Yield: 79\%, Gray Solid, Mp = $123-125^{\circ} \mathrm{C}$; FT-IR, (ATR) $=\bar{v}: 3093(\mathrm{C}-\mathrm{H}$ stretch), 1621<smiles>[X]n1c(-c2cccc(O)c2)nc2cc(Cl)ccc21</smiles>
$\left(\mathrm{C}=\mathrm{N}\right.$ stretch), $1527\left(\mathrm{NO}_{2}\right.$, asym. stretch), 1449 (C=C stretch), $1353\left(\mathrm{NO}_{2}\right.$, sym. stretch), 1195 (C-O stretch), 1101 (C-Cl stretch) $\mathrm{cm}^{-1},{ }^{1} \mathrm{H}$ NMR (400 MHz, Acetone), $\sigma(\mathrm{ppm})=7.9$ (brs, $1 \mathrm{H}_{6}$ ), 7.8 (brs, $1 \mathrm{H}_{7}$ ), 8.0 (brs, $\left.2 \mathrm{H}_{5}{ }^{\prime}, 4\right), 8.5$ (brs, $1 \mathrm{H}_{4}{ }^{\prime}$ ), 8.77 (brs, $1 \mathrm{H}_{6}{ }^{\prime}$ ), 9.0 (brs, $1 \mathrm{H}_{2}$ ); ${ }^{13} \mathrm{CNMR}(300 \mathrm{MHz}, \mathrm{DMSO}), \sigma(\mathrm{ppm})=145.4\left(\mathrm{C}_{2}\right), 128.7\left(\mathrm{C}_{6}{ }^{\prime}\right), 123.8\left(\mathrm{C}_{2}\right), 123.2\left(\mathrm{C}_{6}\right)$, 117.0 $\left(\mathrm{C}_{4}\right), 115.8\left(\mathrm{C}_{7}\right)$.

Yield: 81\%, Gray Solid, Mp = $177-179^{\circ} \mathrm{C}$; FT-IR, $($ ATR $)=\bar{v}: 3066(\mathrm{C}-\mathrm{H}$ stretch $), 1611$ $(\mathrm{C}=\mathrm{N}$ stretch), 1550, $1481(\mathrm{C}=\mathrm{C}$ stretch), 1332 (C-N stretch), 1261 (C-O stretch), 1089 (C-Cl stretch) $\mathrm{cm}^{-1},{ }^{1} \mathrm{H}$ NMR $\left(400 \mathrm{MHz}\right.$, Acetone), $\sigma(\mathrm{ppm})=7.45$ (brs, $\left.1 \mathrm{H}_{6}\right), 7.6-7.8(\mathrm{~m}$, $\left.4 \mathrm{H}_{5}, 6^{\prime}, 3^{\prime}, 2^{\prime}\right), 8.25$ (brs, $\left.2 \mathrm{H}_{4,7}\right) ;{ }^{13} \mathrm{CNMR}$ (300 MHz, DMSO), $\sigma(\mathrm{ppm})=145.4\left(\mathrm{C}_{2}\right), 128.7\left(\mathrm{C}_{5}{ }^{\prime}\right)$, 123.8( $\left(\mathrm{C}_{2}\right), 123.1\left(\mathrm{C}_{6}\right), 117.0\left(\mathrm{C}_{4}\right), 115.8\left(\mathrm{C}_{7}\right) ; \mathrm{C}_{13} \mathrm{H}_{7} \mathrm{Cl}_{2} \mathrm{NO}$, Calculated: $\mathrm{C}, 59.12 ; \mathrm{H}, 2.67$; N, 5.30. Found: C, 58.92; H, 2.45; N, $5.72 \%$.

Yield: $76 \%$, Gray Solid, $\mathrm{Mp}=148-150^{\circ} \mathrm{C}$; FT-IR, $(\mathrm{ATR})=\overline{\mathrm{v}}$ : $3092(\mathrm{C}-\mathrm{H}$ stretch$), 1586$ $(\mathrm{C}=\mathrm{N}$ stretch), 1561, 1448 (C=C stretch), 1388 (C-N stretch), 1257 (C-O stretch), 1092 ${ }^{\mathrm{Cl}}(\mathrm{C}-\mathrm{Cl}$ stretch $) \mathrm{cm}^{-1},{ }^{1} \mathrm{H}$ NMR $\left(400 \mathrm{MHz}, \mathrm{CDCl}_{3}\right), \sigma(\mathrm{ppm})=7.01-7.12\left(\mathrm{~m}, 2 \mathrm{H}_{6,5}\right)^{\circ}, 7.4-7.5$ $\left(\mathrm{m}, 2 \mathrm{H}_{6^{\prime}, 3^{\circ}}\right), 7.81$ (brs, $\left.1 \mathrm{H}_{7}\right), 8.25$ (brs, $\left.1 \mathrm{H}_{4}\right) ;{ }^{13} \mathrm{CNMR}$ (300 MHz, DMSO), $\sigma(\mathrm{ppm})=$ 145.4( $\left(\mathrm{C}_{2}\right), 128.7\left(\mathrm{C}_{6}{ }^{\circ}\right), 123.8\left(\mathrm{C}_{5}{ }^{\prime}\right), 123.1\left(\mathrm{C}_{6}\right), 117.0\left(\mathrm{C}_{4}\right), 115.7\left(\mathrm{C}_{7}\right) ; \mathrm{C}_{13} \mathrm{H}_{6} \mathrm{Cl}_{3} \mathrm{NO}$, Calculated: C, 52.30; H, 2.03; N, 4.69. Found: C, 52.15; H, 2.39; N, $4.48 \%$.

\section{Biological activity}

Microorganisms. The antifungal activities of the synthetic compounds against some standard strains of fungi, including Candida ablicans (ATCC 10261, 1905, 2730, 1912), C. tropicalis (ATCC 4344, 750),
C. krusei (ATCC 6258), C. glabrata (ATCC 90030, 863, 2192), C. dubliniensis (ATCC 8500, 8501, 7988, 7987), C. neoformance (ATCC 9011), Aspergillusflavus (ATCC 64025), A. clavatus (CBS 514.65), A. fumigatus (ATCC 14110) and Exophiala dermatitidis (ATCC 
109136) were determined. In addition, the antifungal activities of the compounds were tested against six clinical isolates of yeasts identified by polymerase chain reaction-restriction fragment length polymorphism (PCR-RFLP) and three clinical isolates of dermatophytes (Epidermophyton floccosum, Microsporum canis and Trichophyton rubrum) identified by both morphological and molecular methods [26]. The antifungal susceptibility of the tested yeasts and Aspergillus species against fluconazole (Sigma, St. Louis, MO, USA) and dermatophytes against griseofulvin (Sigma) were examined by microdilution methods [27, 28].

Determination of minimum inhibitory concentration. MICs were determined by using the broth microdilution method recommended by the CLSI (Clinical \& Laboratory Standards Institute) with some modifications. In order to determinate the antimicrobial activities against fungi, serial dilutions of the synthetic compounds (1 - $1024 \mu \mathrm{g} / \mathrm{mL})$ were prepared in 96-well microtiter plates using Roswell Park Memorial Institute medium (RPMI)-1640 media (Sigma, St. Louis, MO, USA) buffered with 3-(N-morpholino)propanesulfonic acid (MOPS) (Sigma). Stock inoculums were prepared by suspending three colonies of the examined yeast in $5 \mathrm{~mL}$ sterile $0.85 \% \mathrm{NaCl}$, and adjusting the turbidity of the inoculums to $0.5 \mathrm{McFarland}$ standards at $530 \mathrm{~nm}$ wavelengths (this yields stock suspension of $1-5 \times 10^{6}$ cells $/ \mathrm{mL}$ ). For moulds (Aspergillus spp. and dermatophytes), conidia were recovered from the 7-day old cultures grown on potato dextrose agar by a wetting loop with tween-20. The collected conidia were transferred in sterile saline and their turbidity was adjusted to $\mathrm{OD}=0.09-0.11$ that yields $0.4-5 \times 10^{6}$ conidia $/ \mathrm{mL}$. The working suspension was prepared by making a $1 / 50$ and 1/1000 dilution with RPMI of the stock suspension for moulds and yeasts, respectively. Working inoculums $(0.1 \mathrm{~mL})$ were added to the microtiter plates, which were incubated in a humid environment at $30^{\circ} \mathrm{C}$ for $24-48 \mathrm{~h}$. Uninoculated medium $(200 \mu \mathrm{L})$ was included as a sterility control. In addition, growth controls (medium with inoculums but without antibiotics or the synthetic compounds) were also included.

The growth in each well was compared with that of the growth in the control well. MICs were visually determined and defined as the lowest concentration of the compounds produced $\geq 95 \%$ growth reduction compared with the growth in the control well. Each experiment was performed in triplicate.

In addition, media from the wells with fungi showing no visible growth were further cultured on Sabouraud dextrose agar (Merck, Darmstadt, Germany) to determine the minimum fungicidal concentration (MFC). MFCs were determined as the lowest concentration yielding no more than 4 colonies, which resulted in mortality of $98 \%$ of the microbes in the initial inoculums.

SAS map analysis

To perform SAS analysis, the structures of the synthesized compounds were generated using Marvin-
Sketch (Marvin 5.7, 2014, ChemAxon). Subsequently, Open Babel 2.3.2 was used to calculate the MACCS fingerprints of all compounds. The resulted fingerprints were then entered in our in house application implemented in Visual.NET. The pairwise tanimoto distance calculation of compounds was done using the following equation:

$$
T_{d}=\frac{c}{a+b-c},
$$

where (a)and (b) represent the number of bit sets in fingerprint of compared compounds and $c$ denotes the number of common bits [29].

Meanwhile, activity similarities were calculated according to the bellow equation.

$$
\operatorname{sim}_{\text {actitit }}(\mathrm{i}, \mathrm{j})=1-\left(\frac{\left[\text { activity }_{i}-\text { activity }_{j} \mid\right]}{\left[\text { activity }_{\max }-\text { activity }_{\min }\right]}\right) \text {, }
$$

where the two symbols activity max and activity min represent the MIC90 values for the most active and the least potent compounds, respectively [30]. Finally, the visualization of structure activity analysis was performed using a $2 \mathrm{D}$ plot.

\section{Results and Discussion}

Therefore, to optimize the reaction conditions we repeated the reactions in different situations in the point of solvents, catalysts and oxidizing agents (Table II). We have investigated the synthesis of $\mathrm{N}$-2-hydroxyphenyl-4-nitrophenyldimine $(\boldsymbol{a})$ via the condensation of 4-nitrobenzaldehyde (1 mmol) and 2-aminophenol $(1.5 \mathrm{mmol})$ with and without the nano- $\mathrm{TiCl}_{4} \cdot \mathrm{SiO}_{2}$. We observed that the reaction did not continue in the absence of this catalyst (Table II, entry 1). Also we repeated the reaction with different amounts of the catalyst and we found that the most appropriate amount for catalyst was $0.1 \mathrm{~g}$ that gave 2-(4-nitrobenzylideneamino) phenol (imine intermediate) with 97\% yield in $1 \mathrm{~h}$ (Table II, entry 4 ). On the other hand, we tried to repeat the reaction in different solvents such as ethanol, ethylacetate and chloroform (Table II, Entry 3, 6 and 7 respectively). Our results showed that ethanol was the best solvent for this type of reactions (Table II, entry 3 ). In the next step, for oxidizing the imine intermediate to 2-(4-Nitrophenyl)1,3-benzoxazole (c) various reagents were also tested, for example ceric ammonium nitrate (CAN), $p$-chloranil and aerobic oxidation (Table II, entry 9, 10 and 11), but according to our experiments the $\mathrm{KMnO}_{4}$ along with $\mathrm{CH}_{3} \mathrm{COOH}$ by the molar ratio, 1.7:1.4 for $\mathrm{KMnO}_{4}$ and $\mathrm{CH}_{3} \mathrm{COOH}$ respectively was the BEST oxidation agent via grinding for 1 minute (Table II, entry 4).

This procedure for synthesis of benzoxazole was compared with different methods from literature (Table II, entry 12 and 13). Finally 2-aminophenol and its 4-chloro analogue reacted with various aldehydes in the optimized condition as substrates for the synthesis of benzoxazole derivatives and the results being presented in Table III. 


\section{Table II}

Synthesis of N-2-hydroxyphenyl-4-nitrophenyldimine (a) and 2-(4-nitrophenyl)benzo[d]oxazole ( $\boldsymbol{c}$ ) under

\begin{tabular}{|c|c|c|c|c|c|c|c|}
\hline Entry & Catalyst (g) & Oxidant & Solvent & Condition & Time $(\mathrm{h})$ & Yield (\%) & Ref. \\
\hline 1 & No catalyst & $\mathrm{KMnO}_{4} / \mathrm{CH}_{3} \mathrm{COOH}$ & - & RT & 4 & 0 & - \\
\hline 2 & $\mathrm{Nano} \mathrm{TiCl}_{4} \cdot \mathrm{SiO}_{2}(0.15)$ & $\mathrm{KMnO}_{4} / \mathrm{CH}_{3} \mathrm{COOH}$ & - & RT & 3 & 57 & - \\
\hline 3 & Nano-TiCl $4 . \mathrm{SiO}_{2}(0.15)$ & $\mathrm{KMnO}_{4} / \mathrm{CH}_{3} \mathrm{COOH}$ & EtOH & RT & 1 & 92 & - \\
\hline 4 & Nano-TiCl $4 . \mathrm{SiO}_{2}(0.1)$ & $\mathrm{KMnO}_{4} / \mathrm{CH}_{3} \mathrm{COOH}$ & EtOH & RT & 1 & 97 & - \\
\hline 5 & Nano-TiCl $4 . \mathrm{SiO}_{2}(0.05)$ & $\mathrm{KMnO}_{4} / \mathrm{CH}_{3} \mathrm{COOH}$ & EtOH & RT & 3 & 85 & - \\
\hline 6 & Nano- $\mathrm{TiCl}_{4} \cdot \mathrm{SiO}_{2}(0.1)$ & $\mathrm{KMnO}_{4} / \mathrm{CH}_{3} \mathrm{COOH}$ & EtOAc & RT & 1 & 70 & - \\
\hline 7 & Nano-TiCl $4 . \mathrm{SiO}_{2}(0.1)$ & $\mathrm{KMnO}_{4} / \mathrm{CH}_{3} \mathrm{COOH}$ & $\mathrm{CH}_{3} \mathrm{Cl}$ & RT & 3 & 66 & - \\
\hline 8 & Nano-TiCl $4 . \mathrm{SiO}_{2}(0.1)$ & $\mathrm{KMnO}_{4} / \mathrm{CH}_{3} \mathrm{COOH}$ & EtOH & Reflux & 1 & 90 & - \\
\hline 9 & Nano-TiCl $4 . \mathrm{SiO}_{2}(0.1)$ & CAN & EtOH & RT & 1 & 72 & - \\
\hline 10 & Nano-TiCl ${ }_{4} \cdot \mathrm{SiO}_{2}(0.1)$ & $p$-chloranil & EtOH & RT & 1 & 80 & - \\
\hline 11 & Nano- $\mathrm{TiCl}_{4} . \mathrm{SiO}_{2}(0.1)$ & air & $\mathrm{EtOH}$ & RT & 24 & 50 & - \\
\hline 12 & $\mathrm{Ni}-\mathrm{SiO}_{2}$ & - & EtOH & RT & 1.5 & 90 & [22] \\
\hline 13 & Molecular Iodine & - & - & MW & $10 \mathrm{~min}$ & 90 & [23] \\
\hline
\end{tabular}

(a) The molar ratio of 2-aminophenol:4-nitrobenzaldehyde is 1.5:1; RT = room temperature, $\mathrm{MW}=$ microwave

Table III

Preparation of various benzoxazoles in the presence of nano- $\mathrm{TiCl}_{4} \cdot \mathrm{SiO}_{2}{ }^{\mathrm{a}}$

\begin{tabular}{|c|c|c|c|c|c|}
\hline Entry & $\mathbf{R}$ & $\mathbf{R}^{\prime}$ & Products & yield $(\%) b$ & Time (h) \\
\hline $\mathrm{c}$ & $\mathrm{H}$ & 4-NO2 & & 92 & 1 \\
\hline $\mathrm{d}$ & $\mathrm{H}$ & $3-\mathrm{NO} 2$ & & 87 & 1 \\
\hline $\mathrm{e}$ & $\mathrm{H}$ & $2-\mathrm{NO} 2$ & & 90 & 1 \\
\hline $\mathrm{f}$ & $\mathrm{H}$ & $4-\mathrm{Cl}$ & & 85 & 1.5 \\
\hline $\mathrm{g}$ & $\mathrm{H}$ & $2,4-\mathrm{Cl}$ & & 88 & 1.5 \\
\hline $\mathrm{h}$ & $\mathrm{H}$ & $4-\mathrm{Br}$ & & 91 & 1.5 \\
\hline $\mathrm{i}$ & $\mathrm{H}$ & 4-COOCH3 & & 68 & 2.5 \\
\hline $\mathrm{j}$ & $4-\mathrm{Cl}$ & $4-\mathrm{NO} 2$ & & 83 & 2 \\
\hline $\mathrm{k}$ & $4-\mathrm{Cl}$ & $3-\mathrm{NO} 2$ & & 79 & 2 \\
\hline 1 & 4- $\mathrm{Cl}$ & $4-\mathrm{Cl}$ & & 81 & 2 \\
\hline $\mathrm{m}$ & $4-\mathrm{Cl}$ & $2,4-\mathrm{Cl}$ & & 76 & 2 \\
\hline
\end{tabular}

Reaction conditions: 2-aminophenol (1.5 mmol), substituted aldehydes (1 mmol), nano- $\mathrm{TiCl}_{4} \cdot \mathrm{SiO}_{2}(0.1 \mathrm{~g}), \mathrm{RT}$, EtOH. b) Isolated yields 
FARMACIA, 2020, Vol. 68, 1

Table IVa

Minimum inhibitory and fungicidal concentrations of the synthetic compounds $(\mu \mathrm{g} / \mathrm{mL})$ against the examined

fungi

\begin{tabular}{|c|c|c|c|c|c|c|c|c|c|c|c|c|c|c|c|c|c|c|c|}
\hline \multirow{2}{*}{\multicolumn{2}{|c|}{ Microorganism }} & \multicolumn{3}{|c|}{ (c) } & \multicolumn{3}{|c|}{ (d) } & \multicolumn{3}{|c|}{ (e) } & \multicolumn{3}{|c|}{$(f)$} & \multicolumn{3}{|c|}{ (g) } & \multicolumn{3}{|c|}{ (h) } \\
\hline & & 3 & 3 & 3 & 3 & $\frac{3}{2}$ & 3 & 3 & $\frac{3}{2}$ & 3 & 3 & 3 & 3 & 3 & 3 & 3 & 3 & 3 & 3 \\
\hline \multirow{21}{*}{ Yeasts } & C. albicans (ATCC 10261) & 16 & 32 & 512 & 512 & 512 & 512 & 512 & 512 & 512 & 8 & 32 & 512 & 8 & 16 & 512 & 128 & 256 & 512 \\
\hline & C. albicans (ATCC 1905) & 64 & 128 & 512 & 512 & 512 & 512 & 256 & 512 & 512 & 16 & 32 & 512 & 32 & 64 & 512 & 256 & 512 & 512 \\
\hline & C. albicans (ATCC 2730) & 16 & 32 & 512 & 256 & 512 & 512 & 128 & 256 & 512 & 16 & 32 & 512 & 16 & 32 & 512 & 64 & 128 & 512 \\
\hline & C. albicans (ATCC 1912) & 16 & 32 & 512 & 16 & 32 & 512 & 16 & 32 & 512 & 8 & 16 & 512 & 16 & 32 & 512 & 16 & 32 & 512 \\
\hline & C. dubliniensis (ATCC 8500) & 16 & 32 & 512 & 512 & 512 & 512 & 512 & 512 & 512 & 4 & 8 & 512 & 32 & 64 & 512 & 256 & 512 & 512 \\
\hline & C. dubliniensis (ATCC 8501) & 32 & 64 & 512 & 128 & 256 & 512 & 256 & 512 & 512 & 32 & 64 & 512 & 64 & 128 & 512 & 256 & 512 & 512 \\
\hline & C. dubliniensis (ATCC 7988) & 32 & 64 & 512 & 512 & 512 & 512 & 512 & 512 & 512 & 64 & 128 & 512 & 32 & 64 & 512 & 64 & 128 & 512 \\
\hline & C. dubliniensis (ATCC 7987) & 16 & 32 & 512 & 128 & 256 & 512 & 128 & 256 & 512 & 16 & 32 & 512 & 32 & 64 & 512 & 32 & 64 & 512 \\
\hline & C. glabrata (ATCC 90030$)$ & 2 & 4 & 512 & 8 & 16 & 512 & 512 & 512 & 512 & 1 & 2 & 512 & 2 & 4 & 512 & 16 & 32 & 512 \\
\hline & C. glabrata (ATCC 863) & 1 & 2 & 512 & 2 & 4 & 512 & 512 & 512 & 512 & 0.25 & 0.5 & 512 & 0.5 & 1 & 512 & 1 & 2 & 512 \\
\hline & C. glabrata (ATCC 2192) & 2 & 4 & 512 & 8 & 16 & 512 & 64 & 128 & 512 & 1 & 2 & 512 & 4 & 8 & 512 & 8 & 16 & 512 \\
\hline & C. krusei (ATCC 6258) * & 32 & 64 & 512 & 128 & 256 & 512 & 256 & 512 & 512 & 32 & 64 & 512 & 64 & 128 & 512 & 128 & 256 & 512 \\
\hline & C. parapilopsis (ATCC 4344) & 32 & 64 & 512 & 512 & 512 & 512 & 128 & 256 & 512 & 32 & 64 & 512 & 64 & 128 & 512 & 128 & 256 & 512 \\
\hline & C. tropicalis (ATCC 750) & 64 & 128 & 512 & 128 & 256 & 512 & 64 & 128 & 512 & 32 & 64 & 512 & 32 & 64 & 512 & 128 & 256 & 512 \\
\hline & C. tropicalis (SUMSCC 194)* & 128 & 256 & 512 & 512 & 512 & 512 & 512 & 512 & 512 & 64 & 128 & 512 & 128 & 256 & 512 & 512 & 512 & 512 \\
\hline & C. albicans (SUMSCC 239) & 8 & 16 & 512 & 512 & 512 & 512 & 512 & 512 & 512 & 8 & 16 & 512 & 8 & 16 & 512 & 512 & 512 & 512 \\
\hline & C. tropicalis (SUMSCC 611) * & 128 & 256 & 512 & 512 & 512 & 512 & 512 & 512 & 512 & 32 & 64 & 512 & 128 & 256 & 512 & 512 & 512 & 512 \\
\hline & C. tropicalis (SUMSCC 203) & 128 & 256 & 512 & 512 & 512 & 512 & 512 & 512 & 512 & 32 & 64 & 512 & 64 & 128 & 512 & 512 & 512 & 512 \\
\hline & C. tropicalis (SUMSCC 2303)* & 128 & 256 & 512 & 512 & 512 & 512 & 512 & 512 & 512 & 32 & 64 & 512 & 128 & 256 & 512 & 512 & 512 & 512 \\
\hline & C. tropicalis (SUMSCC 625)* & 16 & 32 & 512 & 512 & 512 & 512 & 512 & 512 & 512 & 4 & 8 & 512 & 16 & 32 & 512 & 512 & 512 & 512 \\
\hline & C. neoformans (ATCC 9011) & 64 & 128 & 512 & 512 & 512 & 512 & 512 & 512 & 512 & 32 & 64 & 512 & 64 & 128 & 512 & 128 & 256 & 512 \\
\hline \multirow{7}{*}{ Filamentous fungi } & A. fumigatus (ATCC 14110) & 512 & 512 & 512 & 512 & 512 & 512 & 512 & 512 & 512 & 64 & 128 & 512 & 64 & 128 & 512 & 512 & 512 & 512 \\
\hline & A. flavus (ATCC 64025) & 512 & 512 & 512 & 512 & 512 & 512 & 512 & 512 & 512 & 128 & 256 & 512 & 128 & 512 & 512 & 512 & 512 & 512 \\
\hline & A. clavatus (CBS 514.65) & 512 & 512 & 512 & 512 & 512 & 512 & 512 & 512 & 512 & 64 & 128 & 512 & 64 & 128 & 512 & 512 & 512 & 512 \\
\hline & M. canis (clinical isolate) & 512 & 512 & 512 & 512 & 512 & 512 & 512 & 512 & 512 & 64 & 128 & 512 & 4 & 8 & 512 & 512 & 512 & 512 \\
\hline & T. rubrum (clinical isolate) & 8 & 16 & 512 & 512 & 512 & 512 & 512 & 512 & 512 & 64 & 64 & 512 & 8 & 16 & 512 & 512 & 512 & 512 \\
\hline & E. flucusom (clinical isolate) & 512 & 512 & 512 & 512 & 512 & 512 & 512 & 512 & 512 & 32 & 64 & 256 & 8 & 16 & 64 & 512 & 512 & 512 \\
\hline & E. dermatitidis (ATCC 109136) & 512 & 512 & 512 & 512 & 512 & 512 & 512 & 512 & 512 & 64 & 128 & 512 & 8 & 16 & 512 & 512 & 512 & 512 \\
\hline
\end{tabular}

*Azole-resistant strain; MIC: Minimum inhibitory concentration, MFC: Minimum fungicidal concentration

Table IVb

Minimum inhibitory and fungicidal concentrations of the synthetic compounds $(\mu \mathrm{g} / \mathrm{mL})$ against the examined

\begin{tabular}{|c|c|c|c|c|c|c|c|c|c|c|c|c|c|c|c|c|c|c|c|}
\hline \multirow{2}{*}{\multicolumn{2}{|c|}{ Microorganism }} & \multicolumn{3}{|c|}{ (i) } & \multicolumn{3}{|c|}{ (j) } & \multicolumn{3}{|c|}{ (k) } & \multicolumn{3}{|c|}{ (d) } & \multicolumn{3}{|c|}{ (m) } & \multicolumn{3}{|c|}{ Positive control } \\
\hline & & $\begin{array}{l}3 \\
\text { 乃े } \\
\text { un }\end{array}$ & $\begin{array}{l}3 \\
\overline{8}\end{array}$ & 3. & $\begin{array}{l}3 \\
\overline{2} \\
\text {, }\end{array}$ & $\begin{array}{l}3 \\
\overline{2}\end{array}$ & 3. & $\begin{array}{l}3 \\
\text { בे } \\
\text { on }\end{array}$ & $\begin{array}{l}3 \\
\overline{2}\end{array}$ & 光 & $\begin{array}{l}3 \\
\tilde{n} \\
\tilde{c}\end{array}$ & $\begin{array}{l}3 \\
\text { Зె }\end{array}$ & 光 & $\begin{array}{l}3 \\
\text { กิ } \\
\mathrm{s}_{1}\end{array}$ & 光 & 章 & $\stackrel{3}{3}$ & 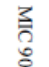 & З \\
\hline \multirow{21}{*}{ Yeasts } & C. albicans (ATCC 10261) & 512 & 512 & 512 & 64 & 128 & 512 & 64 & 128 & 512 & 512 & 512 & 512 & 128 & 256 & 512 & 2 & 8 & 512 \\
\hline & C. albicans (ATCC 1905) & 512 & 512 & 512 & 512 & 512 & 512 & 64 & 128 & 512 & 512 & 512 & 512 & 256 & 512 & 512 & 0.25 & 0.5 & 64 \\
\hline & C. albicans (ATCC 2730) & 256 & 512 & 512 & 32 & 64 & 512 & 32 & 64 & 512 & 256 & 512 & 512 & 16 & 32 & 512 & 0.5 & 1 & 2 \\
\hline & C. albicans (ATCC 1912) & 128 & 256 & 512 & 16 & 32 & 512 & 16 & 32 & 512 & 32 & 64 & 512 & 16 & 32 & 512 & 0.5 & 1 & 64 \\
\hline & C. dubliniensis (ATCC 8500) & 512 & 512 & 512 & 64 & 256 & 512 & 256 & 512 & 512 & 512 & 512 & 512 & 256 & 512 & 512 & 0.25 & 0.5 & 64 \\
\hline & C. dubliniensis (ATCC 8501) & 512 & 512 & 512 & 64 & 128 & 512 & 64 & 128 & 512 & 128 & 256 & 512 & 64 & 128 & 512 & 0.5 & 1 & 32 \\
\hline & C. dubliniensis (ATCC 7988) & 512 & 512 & 512 & 256 & 512 & 512 & 128 & 256 & 512 & 512 & 512 & 512 & 256 & 256 & 512 & 0.5 & 1 & 4 \\
\hline & C. dubliniensis (ATCC 7987) & 512 & 512 & 512 & 64 & 128 & 512 & 64 & 128 & 512 & 64 & 128 & 512 & 32 & 64 & 512 & 0.5 & 1 & 4 \\
\hline & C. glabrata (ATCC 90030$)$ & 512 & 512 & 512 & 8 & 16 & 512 & 8 & 16 & 512 & 512 & 512 & 512 & 512 & 512 & 512 & 2 & 4 & 512 \\
\hline & C. glabrata (ATCC 863) & 512 & 512 & 512 & 2 & 8 & 512 & 0.25 & 0.5 & 512 & 512 & 512 & 512 & 512 & 512 & 512 & 0.5 & 1 & 16 \\
\hline & C. glabrata (ATCC 2192) & 512 & 512 & 512 & 8 & 16 & 512 & 8 & 16 & 512 & 256 & 512 & 512 & 256 & 512 & 512 & 0.25 & 0.5 & 32 \\
\hline & C. krusei (ATCC 6258)* & 512 & 512 & 512 & 128 & 256 & 512 & 256 & 512 & 512 & 512 & 512 & 512 & 128 & 256 & 512 & 32 & 128 & 512 \\
\hline & C. parapilopsis (ATCC 4344) & 512 & 512 & 512 & 32 & 64 & 512 & 32 & 64 & 512 & 128 & 256 & 512 & 32 & 64 & 512 & 1 & 2 & 16 \\
\hline & C. tropicalis (ATCC 750) & 128 & 256 & 512 & 64 & 128 & 512 & 128 & 256 & 512 & 64 & 128 & 512 & 64 & 128 & 512 & 16 & 32 & 512 \\
\hline & C. tropicalis (SUMSCC 194)* & 512 & 512 & 512 & 128 & 256 & 512 & 256 & 512 & 512 & 512 & 512 & 512 & 256 & 512 & 512 & 128 & 256 & 512 \\
\hline & C. albicans (SUMSCC 239) & 512 & 512 & 512 & 256 & 512 & 512 & 512 & 512 & 512 & 256 & 512 & 512 & 512 & 512 & 512 & 0.12 & 0.25 & 512 \\
\hline & C. tropicalis (SUMSCC 611)* & 512 & 512 & 512 & 512 & 512 & 512 & 512 & 512 & 512 & 512 & 512 & 512 & 512 & 512 & 512 & 128 & 256 & 512 \\
\hline & C. tropicalis (SUMSCC 203) & 512 & 512 & 512 & 512 & 512 & 512 & 256 & 512 & 512 & 512 & 512 & 512 & 256 & 512 & 512 & 4 & 8 & 512 \\
\hline & C. tropicalis (SUMSCC 2303)* & 512 & 512 & 512 & 128 & 256 & 512 & 128 & 256 & 512 & 512 & 512 & 512 & 128 & 256 & 512 & 128 & 256 & 512 \\
\hline & C. tropicalis (SUMSCC 625)* & 512 & 512 & 512 & 512 & 512 & 512 & 512 & 512 & 512 & 512 & 512 & 512 & 256 & 512 & 512 & 128 & 256 & 512 \\
\hline & C. neoformans (ATCC 9011) & 512 & 512 & 512 & 512 & 512 & 512 & 32 & 64 & 512 & 512 & 512 & 512 & 128 & 256 & 512 & 0.5 & 1 & 512 \\
\hline \multirow{7}{*}{ Filamentous fungi } & A. fumigatus (ATCC 14110) & 512 & 512 & 512 & 512 & 512 & 512 & 512 & 512 & 512 & 512 & 512 & 512 & 512 & 512 & 512 & 2 & 4 & 512 \\
\hline & A. flavus (ATCC 64025) & 512 & 512 & 512 & 512 & 512 & 512 & 512 & 512 & 512 & 512 & 512 & 512 & 512 & 512 & 512 & 0.5 & 1 & 512 \\
\hline & A. clavatus (CBS 514.65) & 512 & 512 & 512 & 512 & 512 & 512 & 512 & 512 & 512 & 512 & 512 & 512 & 512 & 512 & 512 & 2 & 4 & 512 \\
\hline & M. canis (clinical isolate) & 256 & 512 & 512 & 256 & 512 & 512 & 256 & 512 & 512 & 128 & 512 & 512 & 128 & 256 & 512 & 0.5 & 1 & 512 \\
\hline & T. rubrum (clinical isolate) & 512 & 512 & 512 & 512 & 512 & 512 & 512 & 512 & 512 & 256 & 512 & 512 & 512 & 512 & 512 & 1 & 2 & 512 \\
\hline & E. flucusom (clinical isolate) & 512 & 512 & 512 & 512 & 512 & 512 & 256 & 512 & 512 & 512 & 512 & 512 & 256 & 512 & 512 & 0.5 & 1 & 512 \\
\hline & E. dermatitidis (ATCC 109136) & 512 & 512 & 512 & 512 & 512 & 512 & 512 & 512 & 512 & 512 & 512 & 512 & 256 & 512 & 512 & 8 & 16 & 32 \\
\hline
\end{tabular}

*Azole-resistant strain; MIC: Minimum inhibitory concentration, MFC: Minimum fungicidal concentration

\section{Antifungal activities of the synthetic compounds}

Tables IVa and IVb summarizes the inhibitory activities of the synthetic compounds and control drugs against the tested fungi. By comparing MIC values of the synthetic compounds, $(g)$ and $(f)$ both exhibited strong inhibitory activities against all of the tested fungi including both yeasts and filamentous fungi with MICs GM (geometric means) of $36.2 \mu \mathrm{g} / \mathrm{mL}$ and $46.3 \mu \mathrm{g} / \mathrm{mL}$, respectively. Furthermore, $(\boldsymbol{c})$ inhibited the growth of the both susceptible and resistant strains of Candida at concentrations ranging from $2 \mu \mathrm{g} / \mathrm{mL}$ to $256 \mu \mathrm{g} / \mathrm{mL}$. Although, $(\boldsymbol{k})$ and $(\boldsymbol{j})$ showed no antifungal activities against the examined filamentous fungi, they both inhibited the growth of the tested yeasts, except those of azole-resistant strains. Of the synthetic compound, $(\boldsymbol{f}),(\boldsymbol{k}),(\boldsymbol{c}),(\boldsymbol{g})$ and $(\boldsymbol{m})$ completely inhibited the growth of Crytococccus neoformance at concentrations ranging from $64 \mu \mathrm{g} / \mathrm{mL}$ to $256 \mu \mathrm{g} / \mathrm{mL}$. 
In comparison with the antifungal activities of the synthetic compounds based on variation of substitutions on 2,3 and 4-position of phenyl ring, we found that the compounds $(\boldsymbol{f})$ and $(\boldsymbol{g})$ with $\mathrm{Cl}$ residue in para and ortho-positions of phenyl ring respectively exhibited better antifungal activities against the tested fungi than the other compounds. In comparison to these mentioned compounds, addition of another $\mathrm{Cl}$ residue to the 5-position of benzoxazole ring resulted respectively $(\boldsymbol{l})$ and $(\boldsymbol{m})$ compounds with considerable lower antifungal activities. Replacement of $\mathrm{Cl}$ with $\mathrm{NO}_{2}$ residue in 4-position of phenyl ring of $(\boldsymbol{c})$ reduced its antifungal activity compared to $(f)$.

Similarly, the addition of $\mathrm{Cl}$ residue to the 5-position of benzoxazole ring in $(j)$ reduced its antifungal activity compared to $(\boldsymbol{c})$. Moreover, exchanging of nitro group from para-position in c to ortho or meta-positions in $(\boldsymbol{e})$ and $(\boldsymbol{d})$ considerably decreased its activity on Candida $s p$. Of the examined synthetic compounds $(\boldsymbol{f}),(\boldsymbol{g})$ and $(\boldsymbol{c})$ all were effective against azole-resistant strains of Candida at concentrations ranging from 8 $256 \mu \mathrm{g} / \mathrm{mL}$, suggesting that the modes of action of this compound are different from the examined antibiotics.

Map analysis

The SAS analysis for all species was performed separately. For example the SAS map for Candida albicans is depicted in Figure 1.

STRUCTURE-ACTIVITY LANDSCAPE

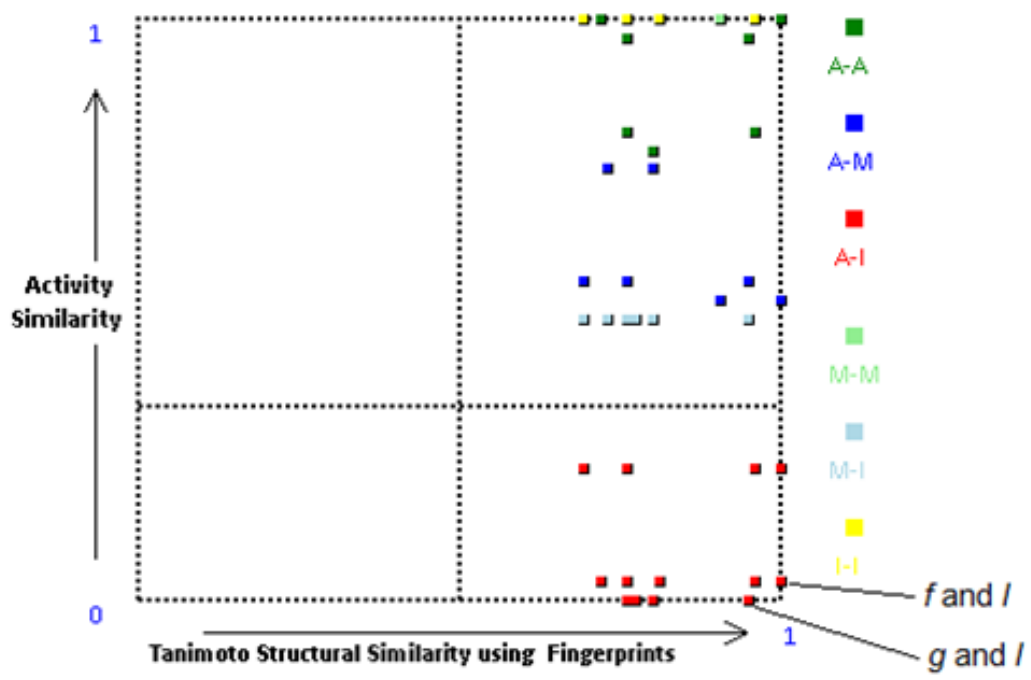

Figure 1.

A plot of SAS-map for C. albicans; where red dots depict "Cliffs", or points which indicate a little difference in structure, but a great difference in activity between the two synthesized substance

As seen in Figure, the SAS plot can be divided into four regions. The bottom right area is presenting the pairwise comparisons of the compounds with similar structures but dissimilar activity values (activity cliffs). As an example an outstanding activity cliff in this series of compounds refers to compounds $(\boldsymbol{f})$ and $(\boldsymbol{l})$ wherein a chlorine atom was inserted at meta-position of benzoxazole ring. This modification led to a significant drop in the activity of the resulted compound $\left((\boldsymbol{f}), \mathrm{MIC}_{50}=8\right.$, and in case of $\left.(\boldsymbol{l}) \mathrm{MIC}_{50}>256\right)$. Also as shown in Figure 1 the two compounds $(\boldsymbol{l})$ and $(\boldsymbol{g})$ are different considering a chlorine atom which is in the 2-phenyl position in compound $(\boldsymbol{g})$ instead of meta-position of benzoxazole in compound $(\boldsymbol{l})$. This difference caused such dissimilarity in effect so that the $\mathrm{MIC}_{50}$ value of compound $(\boldsymbol{l})$ is more than 256 but for compound $(\mathrm{g})$ is 8 .

\section{Conclusions}

In conclusion, we have developed a novel and highly efficient method for the synthesis of benzoxazoles by treatment of 2-aminophenol and substituted aldehydes in the presence of Ni supported silica as an effective Lewis acid. This methodology may find widespread uses in organic synthesis for preparation of the benzoxazoles. In the present study, some of the synthetic compounds including $(f)$ and $(g)$ exhibited a great activity against tested Aspergillus, Candida and dermatophytes. Comparing the structure and activity of these two compounds with the others, revealed that the presence of $\mathrm{Cl}$ residue in para and orthoposition of phenyl ring, enhance the antifungal activity. Altogether, regarding the broad spectrum antifungal activities of some of the tested compounds even against azole resistant strains, they might be good candidates 
for further in vivo studies to elucidate their effects and toxicity as novel antifungal drugs.

\section{Acknowledgement}

Financial assistance from the Shiraz University of Medical Sciences is gratefully acknowledged. The authors would like to thank the Research Consulting Centre (RCC) at Shiraz University of Medical Sciences for their assistance in editing this article.

\section{Conflict of interest}

The authors declare no conflict of interest.

\section{References}

1. Zhao Q, Zou Y, Guo J, Yu S, Chai XY, Hu H, Wu Q, Synthesis and antifungal activities of N-glycosylated derivatives of Tunicyclin $\mathrm{D}$, an antifungal octacyclopeptide. Tetrahedron, 2014; 70: 7780-7787.

2. Acosta P, Insuasty B, Ortiz A, Abonia R, Sortino M, Zacchino SA, Quiroga J, Solvent-free microwaveassisted synthesis of novel pyrazolo $\left[4^{\prime}, 3^{\prime}: 5,6\right]$ pyrido [2,3-d]pyrimidines with potential antifungal activity. Arab J Chem., 2016; 9(3): 481-492.

3. Khabnadideh S, Rezaei Z, Khalafi-Nezhad A, Pakshir K, Heiran MJ, Shobeiri H, Design and synthesis of 2methyl and 2-methyl-4-nitro imidazole derivatives as antifungal agents. Iran J Pharmaceutic Sci., 2009; 5: $31-36$.

4. Khabnadideh S, Rezaei Z, Khalafi-Nezhad A, Pakshir K, Roosta A, Baratzadeh Z, Design and Synthesis of imidazole and benzimidazole derivatives as antifungal agents. Agents Medicin Chem., 2008; 7 : 215-218

5. Rezaei Z, Khabnadideh S, Pakshir K, Hossaini Z, Amiri F, Assadpour E, Design, synthesis, and antifungal activity of triazole and benzotriazole derivatives. Eur J Medicin Chem., 2009; 44: 3064-3067.

6. Khabnadideh S, Rezaei Z, Pakshir K, Zomorodian $\mathrm{K}$, Ghafari N, Synthesis and antifungal activity of benzimidazole, benzotriazole and aminothiazole derivatives. Res Pharmaceut Sci., 2012; 7: 65-72.

7. Arakova $\mathrm{K}$, Inamasu $\mathrm{M}$, Masumoto $\mathrm{M}$, Novel Benzoxazole 2, 4-thiazolidinediones as potent hypoglycemic agents. synthesis and structure-activity relationships. Chem Pharmaceut Bul., 1997; 45: 1984-1993.

8. Kastura Y, Tnoue Y, Nishino S, Studies on antiulcer drugs. III. 1) Synthesis and antiulcer activities of imidazol [1,2-alpha] pyridinylethylbenzoxazoles and related compounds. A novel class of histamine h2-receptor antagonists. Chem Pharmaceut Bul., 1992; 40: 1424-1438.

9. Ismail Y, lkay O, Özlem T, QSARs of some novel isosteric heterocyclics with antifungal activity. Acta Biochimica Polonica, 2000; 47: 481-486.

10. Shrinivasa R, Rao P, Kumar P, Design, synthesis and biological evaluation of benzimidazole/benzothiazole and benzoxazole derivatives as cyclooxygenase inhibitors. Bioorg Medicin Chemy Let., 2003; 13: 657- 660 .
11. Aiello S, Wells G, Ston E, Kadri H, Synthesis and Biological properties of benzothiazole, benzoxazole, and chromen-4-one analogues of the potent antitumor agent 2-(3,4-dimethoxyphenyl)-5-fluorobenzo thiazole (PMX 610, NSC 721648). J Med Chem., 2008; 51: 5135-5139.

12. Sondhi S, Singh N, Kumar A, Lozach O, Synthesis, anti-inflammatory, analgesic and kinase (CDK-1, CDK-5 and GSK-3) inhibition activity evaluation of benzimidazole/benzoxazole derivatives and some Schiff's bases. Bioorg Medicin Chem., 2006; 14: 3758-3765.

13. Altenhoff G, Glorious F, A domino copper-catalyzed $\mathrm{C}[$ bond $] \mathrm{N}$ and $\mathrm{C}[$ bond $] \mathrm{O}$ cross-coupling for the conversion of primary amides into benzoxazoles. Adv Synt Catal., 2004; 346: 1661-1664.

14. Evindar G, Batey RA, Parallel synthesis of a library of benzoxazoles and benzothiazoles using ligandaccelerated copper-catalyzed cyclizations of orthohalobenzanilides. J Org Chem., 2006; 71: 1802-1808.

15. Maddila S, Jonnalagadda SB, Efficient one-pot synthesis of benzoxazole derivatives catalyzed by nickel supported silica. J Chilean Chem Soc., 2012; 57: 1099-1100.

16. Mirjalili BF, Zamani L, Nano-TiCl $4 . \mathrm{SiO}_{2}$ : a versatile and efficient catalyst for synthesis of dihydropyrimidones via Biginelli condensation. South Af J Chem., 2014; 67: 21-26.

17. Zamani L, Mirjalili BF, Synthesis and characterization of tetraarylporphyrins in the presence of nano- $\mathrm{TiCl}_{4} \cdot \mathrm{SiO}_{2}$. Chem Heterocyc Comp., 2015; 51: 578-581.

18. Mirjalili BF, Bamoniri A, Zamani L, Nano- $\mathrm{TiCl}_{4} / \mathrm{SiO}_{2}$ : An efficient and reusable catalyst for the synthesis of tetrahydrobenzo $[a]$ xanthenes-11-ones. Let Org Chem, 2012; 9: 338-343.

19. Zamani L, Mirjalili BF, Zomorodian K, Namazian M, Khabnadideh S, Faghih Mirzaei E, Synthesis of benzimidazoles in the presence of nano- $\mathrm{TiCl}_{4} \cdot \mathrm{SiO}_{2}$ as antifungal agents and tautomerism theoretical study of some products. Farmacia, 2014; 62(3): 467-482.

20. Zamani L, Mirjalili BF, Zomorodian K, Zomorodian S, 'Synthesis and characterization of 5 -substituted $1 \mathrm{H}$ tetrazoles in the presence of nano- $-\mathrm{TiCl}_{4} \cdot \mathrm{SiO}_{2}$. South Afric J Chem., 2015; 68: 133-137.

21. Mirjalili BF, Zamani L, Zomorodian K, Khabnadideh S, Haghighijoo Z, Malakotikhah Z, Ayatollahi Mousavi SA, Khojasteh S, Synthesis, antifungal activity and docking study of 2-amino-4H-benzochromene-3carbonitrile derivatives. J Mol Struct., 2016; 1116: 102-108.

22. Calles JA, Carrero A, Vizcaíno AJ, Lindo M, Effect of $\mathrm{Ce}$ and $\mathrm{Zr}$ Addition to $\mathrm{Ni} / \mathrm{SiO}_{2}$ catalysts for hydrogen production through ethanol steam reforming. Catalysts, 2015; 5: 58-76.

23. Matloubi-Moghaddam F, Bardajee GR, Ismaili H, Dokht-Taimoory SM, Facile and efficient one-pot protocol for the synthesis of benzoxazole and benzothiazole derivatives using molecular iodine as catalyst. Synthetic Communications, 2006; 36: 25432548.

24. Chikhale RV, Pant AM, Menghani SS, Wadibhasme PG, Khedekar PB, Facile and efficient synthesis of benzoxazole derivatives using novel catalytic activity of PEG-SO 3 H. Arab J Chem., 2017; 10(5): 715-725. 
25. Sana T, Sharad W, Synthesis through microwave irradiation, characterization and evaluation of antimicrobial activity of 2-phenyl-1, 3-benzoxazole derivatives. Int Res J Pharm., 2012; 3: 213-217.

26. Mirhendi H, Makimura K, Khoramizadeh M, Yamaguchi $\mathrm{H}$, A One-Enzyme PCR-RFLP assay for identification of six medically important Candida species. Nippon Ishinkin Gakkai Zasshi, 2006; 47: 225-229.

27. Clinical and Laboratory Standards Institute (CLSI), Reference Method for Broth Dilution Antifungal Susceptibility Testing of Yeasts; approved standard, $2^{\text {nd }}$ ed. Wayne, PA: Clin Lab Stand Instit., CLSI M27-A7, 2006.
28. Clinical and Laboratory Standards Institute (CLSI). Reference Method for Broth Dilution Antifungal Susceptibility Testing of Filamentous Fungi; approved Standard, $2^{\text {nd }}$ ed. Wayne, PA: Clin Lab Stand Instit.; CLSI M38-A, 2006.

29. Pérez-Nueno VI, Rabal O, Borrell JI, APIF: a new interaction fingerprint based on atom pairs and its application to virtual screening. J Chem Inform Model., 2009; 49: 1245-1260.

30. Assadieskandar A, Amini M, Salehi M, Sadeghian H, Alimardani M, Sakhteman A, Nadri H, Shafiee A, Synthesis and SAR study of 4,5-diaryl- $1 H$-imidazole2(3H)-thione derivatives, as potent 15-lipoxygenase inhibitors. Bioorg Med Chem, 2012; 20: 7160-7166. 